\title{
Synchronization of Power Networks: Network Reduction and Effective Resistance
}

\author{
Florian Dörfler* Francesco Bullo* \\ * Center for Control, Dynamical Systems, and Computation, \\ University of California at Santa Barbara, Santa Barbara, CA 93106 \\ USA (e-mail: \{dorfler,bullo\}@engineering.ucsb.edu).
}

\begin{abstract}
In transient stability studies in power networks two types of mathematical models are commonly used - the differential-algebraic structure-preserving model and the reduced dynamic model of interconnected swing equations. This paper analyzes the reduction process relating the two power network models. The reduced admittance matrix is obtained by a Schur complement of the topological network admittance matrix with respect to its bus nodes. We provide a detailed spectral, algebraic, and graph-theoretic analysis of this network reduction process, termed Kron reduction, with particular focus on the effective resistance. As an application of this analysis, we are able to state concise conditions relating synchronization in the considered structurepreserving power network model directly to the state, parameters, and topology of the underlying network. In particular, we provide a spectral condition based on the algebraic connectivity of the network and a second condition based on the effective resistance among generators.
\end{abstract}

Keywords: transient stability, synchronization, structure-preserving power network model

\section{INTRODUCTION}

The envisioned future power grid is expected to be extremely complex. Its power generation will be highly distributed and it will rely increasingly on renewable energy sources, such as wind and solar power, which cause stochastic disturbances. In face of these uncertainties and the rising complexity, the detection and rejection of instability mechanisms leading to power blackouts will be one of the major tasks to be handled by the future "smart grid."

One important form of power network stability is transient stability, which is the ability of a power system to remain in synchronism when subjected to large transient disturbances such as faults on system components or significant changes in load or generation. The problem of synchronization and transient stability is well-studied in the power systems community and surveyed by Pai (1989), Alberto et al. (2001), and Chiang (2010). The structure preserving (or network-preserving) power system model considered in transient stability analysis consists of a set of differentialalgebraic equations representing the rotor dynamics of each generator as well as the power flow at each bus. If the loads in the network are modeled as constant impedances, the power system model can be reduced to the well-known swing equations featuring an all-to-all coupling among the generators. This network-reduced model is mathematically tractable but the original network topology representing the system components is lost. Analytic approaches to synchronization in structure-preserving models have been considered by Bergen and Hill (1981), Tsolas et al. (1985), Zou et al. (2003), and Guedes et al. (2005). These approaches rely on Hamiltonian arguments and also lead to computational procedures providing precise estimates of the region of attraction for synchronization. An open problem, recognized by Hill and Chen (2006) and not resolved by classical analysis methods, is the quest for explicit

* This work was supported in part by NSF grants IIS-0904501 and CNS-0834446. and concise conditions for synchronization as a function of state, parameters, and topology of the power network.

In an earlier work the authors analyzed synchronization and transient stability in a network-reduced power system. Among other things, Dörfler and Bullo (2010b) provided a solution to the open problem of relating synchronization in a power network to the underlying network structure. In particular, the synchronization conditions read as "the network connectivity has to dominate the network's nonuniformity (in effective power inputs to the generators) and the network's losses (due to transfer conductances)." Since a network-reduced power system model features allto-all coupling the conditions derived by Dörfler and Bullo (2010b) did not capture the original power network topology. The main contribution of this paper are as follows.

As a first contribution, we provide a rigorous algebraic analysis and graph-theoretic interpretation of the Kron reduction process relating the network-preserving and the network-reduced power system model. In essence, Kron reduction of a network is a Schur complement of the Laplacian matrix with respect to a set of nodes. We relate the spectrum of the resulting Kron-reduced Laplacian matrix to the spectrum of the non-reduced Laplacian matrix and give various interpretations in the spirit of algebraic graph theory. In particular, we relate the elements of the Kronreduced Laplacian to the effective resistance in the nonreduced network, which is a graph-theoretical distance and connectivity measure (Doyle and Snell, 1984). The spectral analysis is presented in detail in Dörfler and Bullo (2010a) whereas this article focuses on the effective resistance. This analysis leads to the second contribution of this paper, the extension of the synchronization conditions derived by Dörfler and Bullo (2010b) to structure-preserving (topological) power network models. The first condition we provide depends on the algebraic connectivity of the nonreduced network, which is a spectral connectivity measure. A second alternative condition depends on the effective resistance among the generators in the non-reduced network. 
These conditions are derived for a lossless network under the assumptions of uniform voltage levels at all generator nodes and zero shunt admittances. For the second condition additionally uniform effective resistances among the generators are assumed, which can be justified for various examples. We are aware that the considered networkpreserving power system model is idealistic, but for this model we can analytically approach the open problem proposed by Hill and Chen (2006): we provide explicit and concise conditions that relate synchronization in a power network to the network state, parameters, and topology.

Paper organization: The remainder of this section introduces some notation. Section 2 recalls the network-preserving and network-reduced power system model as well as the Kron reduction process. Section 3 analyzes the Kron reduction process resulting in the novel synchronization conditions stated in Section 4. Section 5 concludes the paper.

Notation: Given a finite set $\mathcal{Q}$ we let $|\mathcal{Q}|$ be its cardinality and define for $n \in \mathbb{N}$ the index set $\mathcal{I}_{n}:=\{1, \ldots, n\}$. Let 1 and $\mathbf{0}$ be the vectors of unit and zero entries of appropriate dimension, and define $e_{i}$ to be vector of zeros of appropriate dimension with entry 1 at position $i$.

Given a complex-valued 2d-array $\left\{A_{i j}\right\}$ with $i, j \in \mathcal{I}_{n}$, let $A \in \mathbb{C}^{n \times n}$ denote the associated matrix and $A^{*}$ the conjugate transposed matrix, and define $A_{\max }=\max _{i j}\left\{\left|A_{i j}\right|\right\}$ and $A_{\min }=\min _{i j}\left\{\left|A_{i j}\right|\right\}$. We use the following notation (Zhang, 2005): for two non-empty index sets $\alpha, \beta \subseteq \mathcal{I}_{n}$ let $A[\alpha, \beta]$ denote the submatrix of $A$ obtained by the rows indexed by $\alpha$ and the columns indexed by $\beta$ and define the shorthands $A[\alpha, \beta)=A\left[\alpha, \mathcal{I}_{n} \backslash \beta\right], A(\alpha, \beta]=A\left[\mathcal{I}_{n} \backslash \alpha, \beta\right]$, and $A(\alpha, \beta)=A\left[\mathcal{I}_{n} \backslash \alpha, \mathcal{I}_{n} \backslash \beta\right]$. Note the consistency $A[i, j]=A_{i j}$ for $i, j \in \mathcal{I}_{n}$. In case that $A[\alpha, \alpha)$ and $A(\alpha, \alpha]$ are the matrices of zero entries, $A$ is a block-diagonal matrix denoted by $A=\operatorname{blkdiag}(A[\alpha, \alpha], A(\alpha, \alpha))$.

The Schur complement of $A$ w.r.t. $A(\alpha, \alpha)$ is given by

$$
A / A(\alpha, \alpha)=A[\alpha, \alpha]-A[\alpha, \alpha) A(\alpha, \alpha)^{-1} A(\alpha, \alpha]
$$

provided that $A(\alpha, \alpha)$ is nonsingular. If $A$ is Hermitian, then we implicitly assume that its (real) eigenvalues are arranged in increasing order $\lambda_{1}(A) \leq \lambda_{2}(A) \leq \ldots \leq \lambda_{n}(A)$.

For a weighted undirected graph induced by a symmetric and nonnegative adjacency matrix $A=A^{T} \in \mathbb{R}^{n \times n}$, the Laplacian matrix is defined as $L(A)=\operatorname{diag}\left(\sum_{j=1}^{n} A_{i j}\right)-A$ $=L(A)^{T}$. Recall that irreducibility of the Laplacian matrix is equivalent to connectivity of the corresponding graph.

\section{REVIEW OF THE POWER NETWORK MODEL}

This section recalls network-preserving and networkreduced power system models to be found in Pai (1989); Anderson and Fouad (1977); Bergen and Vittal (2000). The models as well as the network reduction process are related to basic matrix and algebraic graph theory.

\subsection{The Network-Preserving Power System Model}

Consider the single-line diagram of a power network $G_{\text {network }}$, such as the New England Power Grid which can be found in Pai (1989) and is schematically illustrated in Figure 1. The nodes of the network can be classified as $n$ generator nodes $\mathcal{V}_{G}, n$ generator terminal buses $\mathcal{V}_{G B}$, and $m$ load buses $\mathcal{V}_{L B}$. The network has the following topology:

(i) each generator node $i_{\mathrm{G}} \in \mathcal{V}_{G}$ is connected to exactly one generator terminal bus $i_{\mathrm{GB}} \in \mathcal{V}_{G B}$,

(ii) each generator terminal bus $i_{\mathrm{GB}} \in \mathcal{V}_{G B}$ is connected to at least one load bus $i_{\mathrm{LB}} \in \mathcal{V}_{L B}$, and

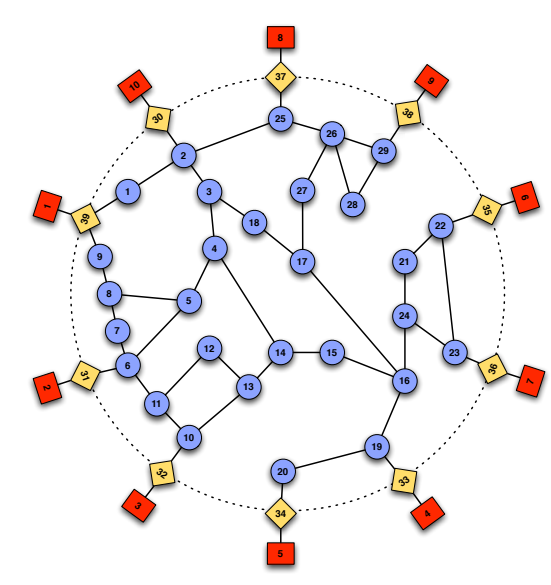

Fig. 1. Schematic representation of the power network topology (i)-(iii) for the New England Power Grid. The symbols $\square, \diamond$, and 0 correspond to the generators $\mathcal{V}_{G}=\{1, \ldots, 10\}$, generator terminal buses $\mathcal{V}_{G B}=\{30-39\}$, and the load buses $\mathcal{V}_{L B}=\{11,29\}$.

(iii) the buses $\mathcal{V}_{G B} \cup \mathcal{V}_{L B}$ form a connected network.

In essence, this topology corresponds to a connected network among the bus nodes $\mathcal{V}_{G B} \cup \mathcal{V}_{L B}$, and the generator nodes $\mathcal{V}_{G}$ are coupled to the interior network via $\mathcal{V}_{G B}$. Adopting nomenclature of circuit theory, the generators and the bus nodes are also denoted as boundary nodes and interior nodes, a distinction which is obvious in Figure 1.

Each edge connecting two nodes $i$ and $j$ is weighted by a non-zero line admittance $Y_{\mathrm{ij}} \in \mathbb{C}$ which is typically of inductive nature, i.e., a negative imaginary part dominates a small positive real part. This weighting of the network $G_{\text {network }}$ gives rise to the complex-valued adjacency matrix

$$
A\left(G_{\text {network }}\right):=\left[\begin{array}{c|c|c}
\mathbf{0} & Y_{\mathrm{G}-\mathrm{GB}} & \mathbf{0} \\
\hline Y_{\mathrm{G}-\mathrm{GB}}^{T} & \mathbf{0} & Y_{\mathrm{GB}-\mathrm{LB}} \\
\hline \mathbf{0} & Y_{\mathrm{GB}-\mathrm{LB}}^{T} & Y_{\mathrm{LB}-\mathrm{LB}}
\end{array}\right] \in \mathbb{C}^{(2 n+m) \times(2 n+m),}
$$

where $Y_{\mathrm{G}-\mathrm{GB}}$ is a permutation of a diagonal matrix (see (i) ), $Y_{\mathrm{GB}-\mathrm{LB}}$ has at least one non-zero entry in every row (see (ii)), and $Y_{\mathrm{LB}-\mathrm{LB}}=Y_{\mathrm{LB}-\mathrm{LB}}^{T}$ is such that the graph among the interior nodes is connected (see (iii)).

Finally, the loads on the network are modeled as passive shunt admittances connecting the buses to the ground:

(iv) each bus $i \in \mathcal{V}_{G B} \cup \mathcal{V}_{L B}$ is connected to the ground via a shunt admittance $Y_{i \text {-ground }}$.

In case the shunt admittance at a bus is zero, the bus is said to be floating. From a viewpoint of circuit theory, the topology (i)-(iv) gives rise to Kirchhoff's equations

$$
I=Y_{\text {network }} V
$$

where $V=\left[V_{\mathrm{G}}\left|V_{\mathrm{GB}}\right| V_{\mathrm{LB}}\right]^{T} \in \mathbb{C}^{2 n+m}$ is the vector of nodal voltages, $\left.I=\left[I_{\mathrm{G}}|\mathbf{0}| \mathbf{0}\right)\right]^{T} \in \mathbb{C}^{2 n+m}$ is the vector of currents injected into the nodes, and $Y_{\text {network }}$ is the $(2 n+$ $m)$-dimensional admittance matrix. The matrix $Y_{\text {network }}$ is the sum of the complex-valued Laplacian $L\left(A\left(G_{\text {network }}\right)\right)=$ $\operatorname{diag}\left(\sum_{j=1}^{n} A\left(G_{\text {network }}\right)_{i j}\right)-A\left(G_{\text {network }}\right)$ and a diagonal matrix containing the shunt admittances:

$$
\begin{aligned}
& Y_{\text {network }}=L\left(A\left(G_{\text {network }}\right)\right) \\
& +\operatorname{blkdiag}\left(\mathbf{0 0}^{T}, \operatorname{diag}\left(Y_{i_{\mathrm{GB}} \text {-ground }}\right), \operatorname{diag}\left(Y_{i_{\mathrm{LB}} \text {-ground }}\right)\right) .
\end{aligned}
$$

In the completely floating case, where all shunt admittances $Y_{i_{\mathrm{GB}} \text {-ground }}$ and $Y_{i_{\mathrm{LB}} \text {-ground }}$ are zero, $Y_{\text {network }}$ is simply a complex-valued Laplacian (or Kirchhoff) matrix. 
The rotor dynamics of generator $i$ are given by the constantvoltage behind reactance model (Anderson and Fouad, 1977)

$$
\frac{M_{i}}{\pi f_{0}} \ddot{\theta}_{i}=-D_{i} \dot{\theta}_{i}+P_{\mathrm{m}, i}-P_{\mathrm{e}, i}, \quad i \in\{1, \ldots, n\},
$$

where the rotor angle $\theta_{i}$ is measured with respect to a rotating frame with frequency $f_{0}, P_{\mathrm{m}, i}>0$ is the mechanical power input, and $M_{i}>0$ and $D_{i}>0$ are the inertia and damping constant. The active output power injected by generator $i$ into the adjacent generator terminal bus (with index $\left.j_{\mathrm{GB}}\right)$ is $P_{\mathrm{e}, i}=\Re\left(V_{i} \cdot V_{j_{\mathrm{GB}}}^{*} Y_{i j_{\mathrm{GB}}}^{*}\right)$. Typically, the loads are modeled as passive shunt admittances. Thus, the passive interior network is governed by Kirchoff's laws and for each bus $i \in \mathcal{V}_{G B} \cup \mathcal{V}_{L B}$ the power flow equations are

$$
0=V_{i} \sum_{j \in \mathcal{V}_{G} \cup \mathcal{V}_{G B} \cup \mathcal{V}_{L B}} Y_{\text {network }}[i j]^{*} V_{j}^{*} .
$$

The generator rotor dynamics (3) and the algebraic power flow equations (4) define the classic differential-algebraic structure-preserving power network model.

\subsection{The Network-Reduced Power System Model}

Since the loads are modeled as passive admittances, all passive nodes $\mathcal{V}_{G B} \cup \mathcal{V}_{L B}$ can be eliminated, i.e., the algebraic equations (4) are removed and the network is reduced to its $n$ active nodes $\mathcal{V}_{\mathrm{G}}$, where the current $I_{\mathrm{G}}$ is injected. Spoken in terms of circuit theory, we look for the reduced admittance matrix $Y_{\text {red }}$ that maps boundary voltages to boundary currents according to $I_{\mathrm{G}}=Y_{\text {red }} V_{\mathrm{G}}$.

Remark 2.1. (Physical interpretation of $\left.Y_{\text {red }}\right)$. The $(i, j)$ element of $Y_{\text {red }}$ can be physically interpreted as the current at a generator node $i$ due to a potential of 1 at the generator node $j$ and 0 at all other generator nodes.

For the subsequent network reduction it is assumed that $Y_{\text {network }}\left(\mathcal{I}_{n}, \mathcal{I}_{n}\right)$ is non-singular, which can be easily verified due to diagonal dominance (as seen in (2)) and irreducibility of $Y_{\text {red }}$ (due to connectivity of the corresponding graph). Standard Gaussian elimination of the lower $n+m$ nodes in equation (1) leads to $I_{\mathrm{G}}=Y_{\text {red }} V_{\mathrm{G}}$, where the reduced admittance matrix $Y_{\text {red }}$ is the Schur complement

$$
Y_{\text {red }}=Y_{\text {network }} / Y\left(\mathcal{I}_{n}, \mathcal{I}_{n}\right) \text {. }
$$

This Schur-complementation is referred to as reduction by structural partitioning in the power systems literature.

Instead of obtaining $Y_{\text {red }}$ as the Schur complement of the suitably partitioned matrix $Y_{\text {network }}$, the interior (passive) nodes $\mathcal{V}_{G B} \cup \mathcal{V}_{L B}$ can be eliminated equivalently by subsequent Kron reduction. Given an admittance matrix $Y \in \mathbb{R}^{k \times k}$, removal of the $k$ th node in $\mathcal{V}_{G B} \cup \mathcal{V}_{L B}$, gives the reduced admittance matrix $Y^{+} \in \mathbb{R}^{(k-1) \times(k-1)}$ as

$$
Y_{i j}^{+}=Y_{i j}-Y_{i k} Y_{j k} / Y_{k k}, \quad i, j \in\{1, \ldots, k-1\} .
$$

Kron's reduction formula (5) corresponds the Schur complement $Y_{\text {network }} / Y_{k k}$, or equivalently, Gaussian elimination of the $k$ th line in equ. (1). The following lemma follows directly from the Quotient Formula (Zhang, 2005, Theorem 1.4) and noting that $Y\left(\mathcal{I}_{n}, \mathcal{I}_{n}\right)$ and $Y_{k k}$ are non-singular.

Lemma 2.2. Subsequent Kron reduction of $Y_{\text {network }}$ with respect to all nodes $\mathcal{V}_{\mathrm{GB}} \cup \mathcal{V}_{\mathrm{LB}}$ is equivalent to the Schur complement $Y_{\text {red }}=Y_{\text {network }} / Y\left(\mathcal{I}_{n}, \mathcal{I}_{n}\right)$.

Subsequent Kron reduction all interior nodes $\mathcal{V}_{\mathrm{GB}} \cup \mathcal{V}_{\mathrm{LB}}$ leads to a complete graph among the boundary nodes $\mathcal{V}_{\mathrm{G}}$, which will be formally shown in the next section. Note also that real (respectively imaginary) matrices $Y_{\text {network }}$ have real (respectively imaginary) reduced matrices $Y_{\text {red }}$.
The off-diagonal elements of $Y_{\text {red }}$ are referred to as transfer admittances and the diagonal elements as self-admittances. Typically, the admittances are inductive on the transmission level, the line admittances dominate the shunt admittances, and $Y_{\text {red }}$ is a fully populated Laplacian-like matrix, which is verified later in Theorem 3.2. For these reasons $Y_{\text {red }}$ typically satisfies $\Re\left(Y_{\text {red }}[i, j]\right) \geq 0$ and $\Im\left(Y_{\text {red }}[i, j]\right)>0$ for all $i \neq j$, and $\Re\left(Y_{\text {red }}[i, i]\right) \geq 0$ and $\Im\left(Y_{\text {red }}[i, i]\right)<0$ for the self-admittances, which we assume from now on.

In the reduced network the electrical output power is $P_{\mathrm{e}, i}=$ $\left.\Re\left(V_{i} \sum_{j=1}^{n} Y_{\text {red }}[i, j]\right)^{*} V_{j}^{*}\right)$ given by the power-angle relation

$$
\begin{aligned}
P_{\mathrm{e}, i}= & \sum_{j=1}^{n}\left|V_{i}\right|\left|V_{j}\right|\left(\Re\left(Y_{\text {red }}[i, j]\right) \cos \left(\theta_{i}-\theta_{j}\right)\right. \\
& \left.+\Im\left(Y_{\text {red }}[i, j]\right) \sin \left(\theta_{i}-\theta_{j}\right)\right) .
\end{aligned}
$$

Equations (3) and (6) give the classic constant-voltage behind reactance model of interconnected swing equations.

\subsection{Review Sufficient Synchronization Conditons}

Consider a lossless power network model with $\Re\left(Y_{\text {red }}[i, j]\right)=$ 0 for all $i, j \in \mathcal{I}_{n}$ and define the coupling weights $P_{i j}:=$ $\left|V_{i}\right|\left|V_{j}\right| \Im\left(Y_{\text {red }}[i, j]\right)>0$ (maximum power transferred between generators $i$ and $j$ ) with $P_{i i}:=0$ for $i \in \mathcal{I}_{n}$. Then the power network model given by (3) and (6) reads as

$$
\frac{M_{i}}{\pi f_{0}} \ddot{\theta}_{i}=-D_{i} \dot{\theta}_{i}+P_{\mathrm{m}, \mathrm{i}}-\sum_{j=1}^{n} P_{i j} \sin \left(\theta_{i}-\theta_{j}\right) .
$$

Consider a weighting of the damping as $\mu=\sum_{k=1}^{n}\left(1 / \prod_{k \neq i}^{n} D_{k}\right)$ $\times \min _{\{i, j\}}\left\{\prod_{k \neq i, j}^{n} D_{k}\right\}\left(\min _{i \neq j}\left\{D_{i} D_{j}\right\} / \max _{i \neq j}\left\{D_{i} D_{j}\right\}\right)^{1 / 2} / n$ (if all $D_{i}$ are equal, then $\mu=1 / D_{i}$ ) and the maximal inertia over damping ratio $\epsilon:=M_{\max } /\left(\pi f_{0} D_{\min }\right)$. Two sufficient conditions for synchronization of the power system model (7) derived by Dörfler and Bullo (2010b) are as follows.

Theorem 2.3. Consider the power network model (7). Assume that either one of the following two conditions hold

$$
\begin{aligned}
n \frac{\min _{i \neq j}\left\{P_{i j}\right\}}{D_{\max }} & >\max _{\{i, j\}}\left\{\frac{P_{\mathrm{m}, \mathrm{i}}}{D_{i}}-\frac{P_{\mathrm{m}, \mathrm{j}}}{D_{j}}\right\}, \\
\lambda_{2}\left(L\left(P_{i j}\right)\right) & >\left\|\left(P_{\mathrm{m}, 2} / D_{2}-P_{\mathrm{m}, 1} / D_{1}, \ldots\right)\right\|_{2} / \mu .
\end{aligned}
$$

If initially all angles $\theta_{i}(0)$ are contained in an arc of length strictly less than $\pi / 2$, then for any bounded initial frequencies $\dot{\theta}_{i}(0)$ there exists $\epsilon^{*}>0$ such that for all $\epsilon<\epsilon^{*}$ the power network model synchronizes exponentially.

Remark 2.4. (Technical comments). It is also possible to give explicit conditions on the asymptotic phase differences, the synchronization frequency and rates, and conditions for phase synchronization. The interested reader is referred to Dörfler and Bullo (2010b). The smallness assumption on $\epsilon$ is a singular perturbation assumption such that the dimension-reduced Kuramoto-type model

$$
D_{i} \dot{\theta}_{i}=P_{\mathrm{m}, i}-\sum_{j=1}^{n} P_{i j} \sin \left(\theta_{i}-\theta_{j}\right), \quad i \in\{1, \ldots, n\} \text {. }
$$

can be analyzed. This assumption is thoroughly justified in Dörfler and Bullo (2010b): among other justifications, the reduced model (10) is (i) topologically equivalent to the full model (7) independent of the magnitude of $\epsilon$, and (ii) equivalent to the model analyzed by the classic and industrially applied PEBS and BCU algorithms (Chiang, 2010).

Remark 2.5. (Physical interpretation of Theorem 2.3) For uniform voltages, $\left|V_{i}\right| \equiv V$, we have that $P_{i j}=V^{2} \Im\left(Y_{\text {red }}[i, j]\right)$ and the left-hand sides of (8)-(9) reflect directly the connectivity of the graph induced by $Y_{\text {red }}$ : the term 
$n \min _{i \neq j}\left\{P_{i j}\right\} / D_{\max }$ is a lower bound for $\min _{i} \sum_{j} P_{i j} / D_{j}$, the worst coupling of one generator to the network, and $\lambda_{2}\left(L\left(P_{i j}\right)\right)$ is the algebraic connectivity of the coupling. The left-hand side measures the non-uniformity in power inputs $P_{\mathrm{m}, \mathrm{i}}$ scaled by the damping $D_{i}$ and the term $\mu$ which captures the non-uniformity in the damping. In summary, conditions (8) and (9) can be interpreted as "the network connectivity has to dominate its non-uniformity."

\section{ANALYSIS OF THE KRON REDUCTION PROCESS}

Consider a real-valued, nonnegative, and irreducible adjacency matrix $A=A^{T} \in \mathbb{R}^{n \times n}$ with zero diagonal elements $A_{i i}=0$ and the corresponding connected and undirected graph. Since $A=-L+\operatorname{diag}\left(\sum_{j \neq i}^{n} L_{i j}\right)$, the Laplacian $L$ equivalently induces the graph. Given a proper subset of nodes $\alpha \subset \mathcal{I}_{n}$ with $2 \leq|\alpha| \leq n-1$, we define the Kronreduced matrix $L_{\mathrm{red}}=L / L(\alpha, \alpha) \in \mathbb{R}^{(n-|\alpha|) \times(n-|\alpha|)}$. For notational simplicity, assume that $\alpha=\{1, \ldots,|\alpha|\}$.

In a lossless power network with purely inductive admittances and zero shunt admittances, the nodes $\alpha$ and $\mathcal{I}_{n} \backslash \alpha$ correspond to the generators and buses, and the matrices $L$ and $L_{\text {red }}$ correspond to $\Im\left(-Y_{\text {network }}\right)$ and $\Im\left(-Y_{\text {red }}\right)$.

It is convenient to regard $L_{\text {red }}$ as obtained by subsequent application of Kron's reduction formula (5). Consider for $\ell \in\{1, \ldots, n-|\alpha|\}$ the following iterative Kron reduction

$$
L^{\ell}=L^{\ell-1} / L_{k_{\ell} k_{\ell}}^{\ell-1}
$$

where $L^{\ell} \in \mathbb{R}^{(n-\ell) \times(n-\ell)}, k_{\ell}=n+1-\ell$ is the lowest diagonal entry of $L^{\ell-1}$, and $L^{0}:=L$ initializes (11). In components, $L^{\ell}$ evolves according to Kron's formula (5):

$$
L_{i j}^{\ell}=L_{i j}^{\ell-1}-L_{i k_{\ell}}^{\ell-1} L_{j k_{\ell}}^{\ell-1} / L_{k_{\ell} k_{\ell}}^{\ell-1}
$$

where $i, j \in\{1, \ldots, n-\ell\}$, By Lemma 2.2, the Kronreduced matrix $L_{\text {red }}$ can be obtained by subsequent reduction of all nodes $k_{\ell} \in \mathcal{I}_{n} \backslash \alpha$, that is, $L / L(\alpha, \alpha)=L^{n-|\alpha|}$. Remark 3.1. (Related literature). In electrical impedance tomography $L_{\text {red }}$ is also referred to as the Dirichlet-toNeumann map (Curtis et al., 1994, 1998). The Schur complement of a matrix and its corresponding graph is also referred to as Schur contraction (Ayazifar, 2002), it is known in the context of (block) Gaussian elimination (Saad, 2003), and serves as an application example in linear algebra (Fan, 2002; Stone and Griffing, 2009).

\subsection{Topological, Algebraic, and Spectral Properties}

This paragraph briefly summarizes some properties established by Dörfler and Bullo (2010a). First, note that the Kron reduction is well-defined, the class of symmetric and irreducible Laplacian matrices is closed under Kron reduction, and the algebraic connectivity is non-decreasing.

Theorem 3.2. (Algebraic and Spectral Properties of Kron Reduction) The following statements hold for the reduced Laplacian matrix $L_{\text {red }}=L / L(\alpha, \alpha)$ :

(1) the Schur complement $L_{\text {red }}=L / L(\alpha, \alpha)$ always exists;

(2) $L_{\text {red }}$ is a symmetric and irreducible Laplacian matrix;

(3) for any $r=1,2, \ldots,|\alpha|$ it holds that

$\lambda_{r}(L) \leq \lambda_{r}\left(L_{\text {red }}\right) \leq \lambda_{r}(L[\alpha, \alpha]) \leq \lambda_{r+n-|\alpha|}(L)$,

in particular it follows that $\lambda_{2}\left(L_{\text {red }}\right) \geq \lambda_{2}(L)$; and

(4) $L_{\text {red }}[i, j] \leq L[\alpha, \alpha][i, j]$ for all $i, j \in \mathcal{I}_{n} \backslash \alpha$.

The next result follows directly from the iterative Kron reduction (12) and the corresponding graph induced by $L^{\ell}$.
Theorem 3.3. (Topological Properties of Kron Reduction). The following statements hold for the graph induced by the Kron-reduced matrix $L_{\text {red }}=L / L(\alpha, \alpha)$ :

(1) All existing edges in the graph induced by $L_{i j}, i, j \in$ $\alpha$, persist in the graph induced by $L_{\text {red }}$;

(2) Kron reduction of the nodes $\mathcal{I}_{n} \backslash \alpha$ leads to a complete graph among all nodes $\alpha$ that were adjacent to the nodes $\mathcal{I}_{n} \backslash \alpha$ prior to the reduction; and

(3) If the nodes $\mathcal{I}_{n} \backslash \alpha$ are connected and each node $\alpha$ is adjacent to at least one node in $\mathcal{I}_{n} \backslash \alpha$, then Kron reduction of all nodes $\mathcal{I}_{n} \backslash \alpha$ leads to a complete graph among the $\alpha$ nodes. Equivalently, the Kron-reduced Laplacian matrix $L_{\text {red }}$ induces a complete graph.

\subsection{Review of the Effective Resistance}

The effective resistance or resistance distance $R_{i j}$ between two nodes $i, j \in \mathcal{I}_{n}$ of an undirected, connected, and uniformly weighted graph with Laplacian $L$ is defined as

$$
R_{i j}:=\left(e_{i}-e_{j}\right)^{T} L^{\dagger}\left(e_{i}-e_{j}\right)=L_{i i}^{\dagger}+L_{j j}^{\dagger}-2 L_{i j}^{\dagger},
$$

where $L^{\dagger}$ is the Moore-Penrose pseudo inverse of $L$. Since $L^{\dagger}$ is symmetric and $R_{i i}:=0$ by definition, the resistance matrix $R$ is again a symmetric matrix. For $i \neq j$ the reciprocal $1 / R_{i j}$ is referred to as the effective conductance. Remark 3.4. (Physical interpretation). If the graph is understood as an electrical network, $R_{i j}$ corresponds to the potential difference between the nodes $i$ and $j$ when a unit current is injected in $i$ and extracted in $j$. Definition (14) can be extended to weighted graphs with additional selfloops if the weights are understood as line conductances and the self-loops as shunt conductances in the circuit.

The effective resistance captures global properties of the graph topology such as distance and connectivity measures. Many interesting results relating $R, L$, and $L^{\dagger}$ can be found in Fouss et al. (2007) and Gutman and Xiao (2004). Applications of effective resistance can be found in the length of random walks (Fouss et al., 2007; Doyle and Snell, 1984), connectivity of biochemical molecules (Klein and Randić, 1993), and distributed estimation algorithms (Barooah and Hespanha, 2009). In the literature on electrical impedance tomography the effective resistance is sometimes associated with the Dirichlet-to-Neumann map, i.e., the reduced Laplacian $L_{\text {red }}$ (Curtis et al., 1994; Saksena, 2002; Ehrlich, 1996; Jorgensen and Pearse, 2009).

There are other methods to compute the effective resistance, for example, via an auxiliary Laplacian with self-loops (Gutman and Xiao, 2004) or via the grounded Laplacian $L(n, n)$, where the removed node $n$ is taken as reference (Barooah and Hespanha, 2009; Saksena, 2002; Ayazifar, 2002). The intuition in Remark 3.4 implies that the effective resistance $R_{i j}$ equals again the potential difference between $i$ and $j$. This intuition can be confirmed.

Lemma 3.5. For all $i, j \in \mathcal{I}_{n}$ and $\gamma \neq 0$ it holds that

$$
R_{i j} \equiv\left(e_{i}-e_{j}\right)^{T}\left(L-(\gamma / n) \mathbf{1}^{T} \mathbf{1}\right)^{-1}\left(e_{i}-e_{j}\right) .
$$

For all $i, j \in \mathcal{I}_{n-1}$ it holds that

$$
R_{i j} \equiv\left(e_{i}-e_{j}\right)^{T} L(n, n)^{-1}\left(e_{i}-e_{j}\right) .
$$

Proof. The identity (15) is a simple generalization of Theorem 5 in Gutman and Xiao (2004). To prove (16), we define $\hat{L}:=L(n, n)$. From (Fouss et al., 2007, Appendix B, eq. (17)) it holds that $\hat{L}_{i j}^{-1}=L_{i j}^{\dagger}-L_{i n}^{\dagger}-L_{j n}^{\dagger}-L_{n n}^{\dagger}$. The identity (16) can now be directly verified. 
Remark 3.6. In (16) the reference node $n$ is chosen arbitrarily. By choosing a different reference node, say 1, all elements $R_{i n}, i \in\{2, \ldots, n\}$, can be obtained from $L(1)$. $\square$

\subsection{Relationship between the Reduced Laplacian Matrix and the Matrix of Effective Resistances}

The intuition in the Remarks 2.1 and 3.4 suggests that the elements of the reduced Laplacian $L_{\text {red }}[i, j]$ are related to the corresponding effective conductances $1 / R_{i j}$. The following theorem gives the exact relation between the reduced Laplacian matrix and the corresponding effective resistances. In essence, the effective resistance among the $\alpha$ nodes is invariant under Kron reduction of the nodes $\mathcal{I}_{n} \backslash \alpha$. Theorem 3.7. (Invariance of Effective Resistance) Consider the reduced Laplacian $L_{\mathrm{red}}=L / L(\alpha, \alpha)$ and the matrix $R$ of effective resistances as defined in (14). Then for $i, j \in \alpha, i \neq j$, it holds that $R_{i j}=\left(e_{i}-e_{j}\right)^{T} L_{\text {red }}^{\dagger}\left(e_{i}-e_{j}\right) . \square$

Proof. Let $\delta>0$ and consider the modified and nonsingular Laplacian $\tilde{L}:=L+(\delta / n) \mathbf{1 1}^{T}$ and its inverse given by $L^{\dagger}+(1 / \delta n) \mathbf{1 1}^{T}$ (Gutman and Xiao, 2004, Generalization of Theorem 5). In analogy to Lemma 3.5, it holds that

$$
\begin{aligned}
R_{i j}=\left(e_{i}-e_{j}\right)^{T}\left(L^{\dagger}+\right. & \left.(1 / \delta n) \mathbf{1 1}^{T}\right)\left(e_{i}-e_{j}\right) \\
& =\left(e_{i}-e_{j}\right)^{T} \tilde{L}^{-1}\left(e_{i}-e_{j}\right)
\end{aligned}
$$

since $\left(e_{i}-e_{j}\right)^{T} \mathbf{1}=\mathbf{0}$. Note that we are only interested in the effective resistances among the nodes $\alpha$, i.e., the $|\alpha| \times|\alpha|$ block of $\tilde{L}^{-1}$. The Schur complement formula (Zhang, 2005, Theorem 1.2) gives the $|\alpha| \times|\alpha|$ block of $\tilde{L}^{-1}$ as $(\tilde{L} / \tilde{L}(\alpha, \alpha))^{-1}$. Consequently, (17) is rendered to

$$
R_{i j}=\left(e_{i}-e_{j}\right)^{T}(\tilde{L} / \tilde{L}(\alpha, \alpha))^{-1}\left(e_{i}-e_{j}\right)
$$

Note that the right-hand side of (17), or equivalently (18), is independent of $\delta$ since the matrices are evaluated on the subspace orthogonal to $\mathbf{1}$, the nullspace of $\tilde{L}$ as $\delta \downarrow 0$. Thus, on the image of $L$ the limit of the right-hand side of (18) exists as $\delta \downarrow 0$. By definition, $L^{\dagger}$ acts as regular inverse on the image of $L$, and equation (18) is rendered to $R_{i j}=\left(e_{i}-e_{j}\right)^{T}(L / L(\alpha, \alpha))^{\dagger}\left(e_{i}-e_{j}\right)$. Finally, recall that $L_{\mathrm{red}}=L / L(\alpha, \alpha)$ which yields the claimed identity. $\square$

Theorem 3.7 establishes a simple relationship between the matrices $R$ and $L_{\text {red }}^{\dagger}$. Other methods constructing $R$ from $L_{\text {red }}^{\dagger}$ can be found in (Curtis et al., 1994; Saksena, 2002; Ehrlich, 1996). An implicit relation without pseudo inverse is given by the Penrose equation $L_{\mathrm{red}} R[\alpha, \alpha] L_{\mathrm{red}}=-2 L_{\mathrm{red}}$ which can be derived from (Xiao and Gutman, 2003, Theorem 6). In general, it is not possible to derive an explicit algebraic relationship between $R$ and $L_{\text {red }}$ or relate bounds on $R$ to bounds on $L_{\text {red }}$. This is not surprising since the general problem of element-wise bounding inverses of interval matrices is known to be NP-hard. However, an analytical relationship between $R$ and $L_{\text {red }}$ can be found if the resistances among the nodes $\alpha$ are uniform.

Corollary 3.8. The following statements are equivalent:

(1) the off-diagonal elements of $L_{\text {red }}$ are uniform, i.e., there is $\lambda>0$ such that $L_{\mathrm{red}}[i, j]=-\lambda$ for all $i, j \in \mathcal{I}_{|\alpha|}, i \neq j$;

(2) the effective resistance $R_{i j}$ among the nodes $\alpha$ is uniform, i.e., there is $r>0$ such that $R_{i j}=r$ for all $i, j \in \alpha, i \neq j$.

Moreover, if both cases are true, then $\lambda=(2 /|\alpha|) / r$.
Proof. Assume 1) holds. Hence, $L_{\text {red }}=\lambda\left(|\alpha| I_{|\alpha|}-\mathbf{1 1}^{T}\right)$ and $L_{\text {red }}^{\dagger}$ is readily obtained as $L_{\text {red }}^{\dagger}=\left(|\alpha| I_{|\alpha|}-\mathbf{1 1}^{T}\right) /\left(|\alpha|^{2} \lambda\right)$. This can be easily verified since $L_{\text {red }}^{\dagger}$ satisfies the four Penrose equations. According to Theorem 3.7, we obtain the effective resistance $R_{i j}=2 /(|\alpha| \lambda)=r$ for $i, j \in \alpha, i \neq j$.

Assume that 2) holds. According to Theorem 3.7 this is equivalent to the $m:=|\alpha|(|\alpha|-1) / 2$ linear equations

$$
r=-\sum_{k \neq i}^{|\alpha|} L_{\text {red }}^{\dagger}[i, k]-\sum_{k \neq j}^{|\alpha|} L_{\text {red }}^{\dagger}[j, k]-2 L_{\text {red }}^{\dagger}[i, j]
$$

for $m$ unknowns $L_{\text {red }}^{\dagger}[i, j]$ (the diagonal resistances are obtained as $\left.L_{\text {red }}^{\dagger}[i, i]=-\sum_{k \neq i}^{|\alpha|} L_{\text {red }}^{\dagger}[i, k]\right)$. One solution to (19) is obviously given by the uniform solution $L_{\text {red }}^{\dagger}[i, j]=$ $-r /(2|\alpha|)$ for all $i, j \in \mathcal{I}_{|\alpha|}, i \neq j$. This solution is isolated since $q \cdot(-r /(2|\alpha|))$ is a solution to (19) iff $q=1$. Thus, the elements $L_{\text {red }}^{\dagger}[i, j]=-r /(2|\alpha|)$ and the corresponding elements $L_{\text {red }}[i, j]=-2 /(r|\alpha|)$ are uniform for $i, j \in \alpha, i \neq j$. $\square$

The following examples demonstrate that uniform resistances among a set of nodes occur for various graph topologies, where we assumed uniform weightings for simplicity. Example 3.9. (Uniform Effective Resistances). In the trivial case, $|\alpha|=2$, Corollary 3.8 reduces to (Jorgensen and Pearse, 2009, Corollary 4.41) and the effective resistance among the $\alpha$ nodes is clearly uniform. Second, if the $\alpha$ nodes are 1-connected leaves of a highly symmetric graph among the nodes $\mathcal{I}_{n} \backslash \alpha$, such as a star-shaped tree, a complete graph, or a combination of these two, then the effective resistance among the $\alpha$ nodes is uniform. Third, the effective resistance in large-scale small-world networks is known to become uniform among sufficiently distant nodes (Korniss et al., 2006). Fourth, with increasing number of nodes the effective resistance in random geometric graphs converges to a degree-dependent limit (Radl et al., 2009 ), which is uniform for various geometries and node distributions. Fifth and finally, geometric graphs such as lattices and their fuzzes are special random geometric graphs with vertices sampled on a grid. According to the previous arguments, the resistance among sufficiently distant lattice nodes becomes uniform in the large limit. $\square$

\section{SPECTRAL AND RESISTANCE-BASED CONDITIONS FOR SYNCHRONIZATION}

In the following, the results of Section 3 will be applied to a lossless power network, where $Y_{\text {network }}$ is purely inductive. We assume uniform voltages $\left|V_{i}\right|=V$ and that the shunt admittances can be modeled equivalently as admittances with respect to an auxiliary reference bus, and thus all buses are floating. In this case, $\Im\left(-Y_{\text {network }}\right)$ is a real-valued Laplacian and it follows that $\Im\left(-Y_{\text {red }}\right)=L\left(P_{i j}\right) / V^{2}$

One of the following two synchronization conditions requires uniform effective resistances among the generators $\mathcal{V}_{\mathrm{G}}$. Note that this assumption is different from requiring uniform line admittances. This assumption can be verified for Examples 3.9 and is also reasonable from a physical viewpoint: the generators are spread over the network such that they can effectively balance the loads. Thus, the potential difference (the effective resistance) should ideally be equal for all generator pairs. Under this assumption we can state the following corollary to Theorem 2.3.

Corollary 4.1. (Spectral and Resistance-based Synchronization Condition) Consider the reduced power network model (7) derived from $G_{\text {network }}$ with floating buses, and 
assume uniform voltages $\left|V_{i}\right|=V$ for all generators. Assume that either one of the two following conditions hold:

(i) the effective conductance $1 / R$ among all generator nodes in $G_{\text {network }}$ is uniform and larger than a critical value, i.e.,

$$
\frac{1}{R}>\max _{\{i, j\}}\left\{\frac{P_{\mathrm{m}, \mathrm{i}}}{D_{i}}-\frac{P_{\mathrm{m}, \mathrm{j}}}{D_{j}}\right\} \frac{D_{\max }}{2 V^{2}},
$$

or (ii) the algebraic connectivity of the power network $G_{\text {network }}$ is larger than a critical value, i.e.,

$\left.\lambda_{2}\left(\Im\left(-Y_{\text {network }}\right)\right)\right)>\left\|\left(P_{\mathrm{m}, 2} / D_{2}-P_{\mathrm{m}, 1} / D_{1}, \ldots\right)\right\|_{2} /\left(V^{2} \mu\right)$.

If initially all angles $\theta_{i}(0)$ are contained in an arc of length strictly less than $\pi / 2$, then for any bounded initial frequencies $\dot{\theta}_{i}(0)$ there exists $\epsilon^{*}>0$ such that for all $\epsilon<\epsilon^{*}$ the power network model synchronizes exponentially.

Proof. Under the assumptions in case (i), it follows from Corollary 3.8 that $\left|Y_{\text {red }}[i, j]\right|=2 /(n R)$ and consequently also $\min _{i \neq j}\left\{P_{i j}\right\}=2 V^{2} /(n R)$. Thus condition (8) in Theorem 2.3 is rendered to (20). In case (ii), condition (21) guarantees condition (9) in Theorem 2.3 due to Theorem 3.2. Synchronization follows directly from Theorem 2.3. $\square$

\section{CONCLUSIONS}

This paper studied synchronization in a simple networkpreserving power system model. In particular, the network reduction to the swing equations model was related to the reduced Laplacian matrix for which various algebraic and graph-theoretic properties were established, in particular the relationship to the effective resistance. These results allowed the extension earlier synchronization conditions by Dörfler and Bullo (2010b) for network-reduced power system models to network-preserving models.

In order to state the final synchronization conditions, various assumptions have to be made on the power network side. The following assumptions should be removed to render the power network model more realistic: purely inductive line admittances, non-zero shunt admittances, and non-uniform voltages during transients. The authors ongoing research addresses sharper synchronization conditions, the effects of loads modeled as shunt admittances, and further properties of the Kron reduction process.

\section{REFERENCES}

Alberto, L.F.C., Silva, F.H.J.R., and Bretas, N.G. (2001). Direct methods for transient stability analysis in power systems: state of art and future perspectives. In IEEE Power Tech Proceedings. Porto, Portugal.

Anderson, P.M. and Fouad, A.A. (1977). Power System Control and Stability. Iowa State University Press.

Ayazifar, B. (2002). Graph Spectra and Modal Dynamics of Oscillatory Networks. Ph.D. thesis, Massachusetts Institute of Technology.

Barooah, P. and Hespanha, J.P. (2009). Error scaling laws for linear optimal estimation from relative measurements. IEEE Transactions on Information Theory, 55, 5661-5673.

Bergen, A.R. and Hill, D.J. (1981). A structure preserving model for power system stability analysis. IEEE Transactions on Power Apparatus and Systems, 100(1), 25-35.

Bergen, A.R. and Vittal, V. (2000). Power Systems Analysis. Prentice Hall, 2 edition.

Chiang, H.D. (2010). Direct Methods for Stability Analysis of Electric Power Systems. Wiley.

Curtis, E., Mooers, E., and Morrow, J. (1994). Finding the conductors in circular networks from boundary measurements.
RAIRO - Modélisation Mathématique et Analyse Numérique, 28(7), 781-814.

Curtis, E., Ingerman, D., and Morrow, J. (1998). Circular planar graphs and resistor networks. Linear Algebra and its Applications, 283(1-3), 115-150.

Dörfler, F. and Bullo, F. (2010a). Spectral analysis of synchronization in a lossless structure-preserving power network model. In IEEE International Conference on Smart Grid Communications. Gaithersburg, MD. Submitted.

Dörfler, F. and Bullo, F. (2010b). Synchronization and transient stability in power networks and non-uniform Kuramoto oscillators. IEEE Transactions on Automatic Control. Submitted.

Doyle, P.G. and Snell, J.L. (1984). Random Walks and Electric Networks. Mathematical Association of America.

Ehrlich, A.M. (1996). Parallel and series addition of networks. URL http://math. washington.edu.

Fan, Y. (2002). Schur complements and its applications to symmetric nonnegative and $Z$-matrices. Linear Algebra and its Applications, 353(1-3), 289-307.

Fouss, F., Pirotte, A., Renders, J.M., and Saerens, M. (2007). Random-walk computation of similarities between nodes of a graph with application to collaborative recommendation. IEEE Transactions on Knowledge and Data Engineering, 19(3), 355-369.

Guedes, R.B.L., Silva, F., Alberto, L.F.C., and Bretas, N.G. (2005). Large disturbance voltage stability assessment using extended Lyapunov function and considering voltage dependent active loads. In IEEE Power Engineering Society General Meeting, 1760-1767. San Francisco, CA.

Gutman, I. and Xiao, W. (2004). Generalized inverse of the Laplacian matrix and some applications. Bulletin: Classe des Sciences Mathématiques et Natturalles, Sciences Mathématiques, 129(29), 15-23.

Hill, D.J. and Chen, G. (2006). Power systems as dynamic networks. In IEEE Int. Symposium on Circuits and Systems, 722-725. Kos, Greece.

Jorgensen, P.E.T. and Pearse, E.P.J. (2009). Operator theory of electrical resistance networks. Available at http://arxiv.org/abs/0806.3881.

Klein, D.J. and Randić, M. (1993). Resistance distance. Journal of Mathematical Chemistry, 12(1), 81-95.

Korniss, G., Hastings, M.B., Bassler, K.E., Berryman, M.J., Kozma, B., and Abbott, D. (2006). Scaling in small-world resistor networks. Physics Letters A, 350(5-6), 324-330.

Pai, M.A. (1989). Energy Function Analysis for Power System Stability. Kluwer Academic Publishers.

Radl, A., von Luxburg, U., and Hein, M. (2009). The resistance distance is meaningless for large random geometric graphs. In Workshop on Analyzing Networks and Learning with Graphs. Whistler, B.C., Canada.

Saad, Y. (2003). Iterative Methods for Sparse Linear Systems. SIAM.

Saksena, N. (2002). The Dirichlet problem for infinite networks. URL http://math. washington.edu.

Stone, E. and Griffing, A. (2009). On the Fiedler vectors of graphs that arise from trees by Schur complementation of the Laplacian. Linear Algebra and its Applications, 431(10), 1869-1880.

Tsolas, N., Arapostathis, A., and Varaiya, P. (1985). A structure preserving energy function for power system transient stability analysis. IEEE Transactions on Circuits and Systems, 32(10), 1041-1049.

Xiao, W. and Gutman, I. (2003). Resistance distance and Laplacian spectrum. Theoretical Chemistry Accounts, 110(4), 284-289.

Zhang, F. (2005). The Schur Complement and its Applications. Springer.

Zou, Y., Yin, M.H., and Chiang, H.D. (2003). Theoretical foundation of the controlling UEP method for direct transientstability analysis of network-preserving power system models. IEEE Transactions on Circuits and Systems I: Fundamental Theory and Applications, 50(10), 1324-1336. 